\title{
Connectionist Natural Language Parsing
}

DOMINIC PALMER-BROWN ${ }^{\mathrm{a}, *}$, JONATHAN A. TEPPER ${ }^{\mathrm{b}}$ and HEATHER M. POWELL ${ }^{\mathrm{b}}$

${ }^{a}$ Leeds Metropolitan University, Computational Intelligence Research Group, School of Computing, Beckett Park, Leeds, LS6 3QS, UK. (d.palmer-brown@lmu.ac.uk)

${ }^{b}$ The Nottingham Trent University, Department of Computing \& Mathematics, Burton Street, Nottingham, NG1 $4 B U, U K$

Teaser: The extent to which connectionist systems have succeeded in parsing a wide range of realistic sentences, containing syntactic structures that are commonly found in natural language interaction, is reviewed, and an assessment is made of their ability to model human sentence processing.

Keywords: connectionism, neural networks, parsing, syntactic processing, corpus.

\section{Summary}

The key developments of two decades of connectionist parsing are reviewed. Connectionist parsers are assessed according to their ability to automatically learn from examples to represent syntactic structures, without being presented with symbolic grammar rules. This review also considers the extent to which connectionist parsers offer computational models of human sentence processing and provide plausible accounts of psycholinguistic data. In considering these issues, special attention is paid to the level of realism, the nature of the modularity, and the type of processing that is to be found in a wide range of parsers.

\section{Introduction}

Connectionist parsers are neural network based systems (Boxes 1 and 2) designed to process words or their syntactic types (tags) in order to produce a correct syntactic 
interpretation, or parse, of complete sentences. Parsers vary greatly in the way in which they tackle syntactic processing, and this is reflected in their modularity (or non-modularity) and in whether they combine neural networks with conventional symbolic processing to provide a hybrid solution, or adopt a purely connectionist approach.

Modularity and hybridity are reviewed as key attributes of connectionist parsers concerned with how the parsing problem is decomposed into (usually simpler) modules to form a parsing system, consisting of one or more connectionist modules and zero or more non-connectionist (e.g. symbolic) modules. The level of realism of parsers is assessed, by which we mean the ability of connectionist parsing systems to capture naturally occurring linguistic structures, behaviours and processing limitations. We discuss the extent to which parsers are able to capture the syntactic constraints and structures that naturally occur in language, as opposed to being limited to artificial grammars that restrict them to processing very small sub-domains of the language.

\section{Modularity and Hybridity}

Psycholinguistic and fMRI-based evidence suggests that there is a significant component of purely syntactic processing of language that precedes and is independent of semantic processing [1]. This evidence can be taken as support for a syntactic module in a Fodorian sense [2], in that it is consistent with information encapsulation and spatial separation of processes. However, there is little consensus in terms of the details of how the syntactic module and its processing might be decomposed into 
separate modules performing distinct tasks. Within the connectionist research programme, many versions of modular architecture have been proposed, and since the neurocognitive evidence is as yet unclear, connectionists are free to explore the computational plausibility of different architectures. When a proposed architecture shows humanlike performance on some aspect of syntax, it may be claimed as evidence of cognitive plausibility, but thus far no systems have been extended to a truly convincing range of language structures. What is clear, however, is that modular and hybrid parsers trained on corpora continue to make significant progress. Indeed, for large scale parsing, whilst there is little support for the eliminative connectionist viewpoint, which claims that purely connectionist systems are in principle capable of cognitive functions including language processing, there is some support for those such as Pinker and Marcus [3] who point out that essentially symbolic processing devices such as registers need to be combined with neural networks in systems such as parsers.

Singular architectures

A common, singular (non-modular) localist (see Boxes 1 and 2) approach has been to explicitly represent the syntactic structure (or parse tree) of a sentence by patterns of activation between layers of units. The units represent symbols or concepts drawn from either symbolic grammar rules [4,5,6,7], word senses, syntactic roles or semantic roles $[8,9,10]$. Many distributed parsing models employing a single neural network have also been developed, using either an FF-MLP network [11,12], an SRN [13,14] or a fully recurrent network [15]. To process linguistic input, which is sequential by nature, FFMLPs (Box 1) typically require a temporal (or sliding) input window of sufficient width, i.e. with a sufficient number of input units, to process the largest grouping of 
constituent symbols that could signify a parser action. This approach is inefficient as the window width is defined by the worst (longest) case, and for all other cases there is redundancy involved in processing the entire window. These limitations were reflected in early distributed connectionist parsers with temporal input windows [11,12].

The Simple Recurrent Network (SRN)

SRN-based architectures (Box 1) belong to the class of recurrent neural networks (RNNs) that implement temporal processing with feedback connections which allow them to establish temporal relationships across sequential input items. SRNs are computationally more powerful than FF-MLP networks for automatically inducing grammatical constraints and structure through supervised learning $[13,14,16]$. For example, SRNs (and RNNs generally) are able to learn regular languages by forming a continuously-valued representation of a corresponding discrete finite-state machine (FSM) when trained to predict the next symbol (eg. word) within a sequence (e.g. sentence) [13, 17]. However, it is generally accepted that natural languages are not strictly regular languages but actually lie between the classes of context-free and context-sensitive languages [18]. This would suggest that additional external apparatus, such as symbolic or neural stacks or registers, are required to extend SRNs to non-regular languages that support embedded and contextsensitive structures. Some have indeed used SRNs with external stacks for language parsing [19-24], whilst others have used alternative RNNs with external stack memories [25].

Rather than introducing additional apparatus to process natural language, a number of researchers have focused their attention on using dynamical systems theory [26] to 
understand and interpret the representations formed and dynamic behaviours (transitions made between hidden states) exhibited by RNNs when they are expected to process irregular languages. Such studies have shown that SRNs learn simple counting functions to process deterministic context-free languages [27]. More recently it has been shown that SRNs are able to generalise to a subset of context-sensitive languages beyond the training data [28], however performance degrades rapidly with respect to the length of the input. Although these studies also show that the behaviour of SRNs is unstable with respect to complexities associated with irregular languages, SRNs are able to generalise to structures considered beyond the computational power of an FSM and the memory limitations exhibited have been shown to be comparable to human memory limitations during sentence processing.

Modules

The first modular distributed parsers appeared in the early 1990's and typically consisted of combinations of FF-MLP, SRN and RAAM architectures. The motivation to decompose the parsing task into sub-modules is often a) to simplify the network's learning task, and to reduce training set size and complexity; and/or b) to evaluate the computational and cognitive plausibility of a given composition of modules. The wide range of modules that has been tried reflects the respective strengths and weaknesses of FF-MLPs, SRNs, and RAAMs. FF-MLPs are general purpose pattern recognisers and function approximators, and therefore it is unsurprising that they have been used to compress [20], transform [29] and compare patterns representing words, phrases, clauses, and semantic and syntactic roles [30]. SRN's, by virtue of sequential input and feedback to context nodes, are more suited than FF-MLPs to recognising a sequence or identifying an 
action based on a sequence, where the sequence is presented incrementally through time. Thus, SRNs lend themselves naturally to segmenting sentences into case-roles [31,21] and phrases [30,32], to signifying shift-reduce actions [22], and to recognising phrases through time $[23,24,30]$. RAAMs, because of their autoassociative coding and decoding properites, have been used to encode (storing them in a compressed form) and decode symbolic parse trees and phrases [34,21,23,35]. Neural networks developments in the form of FF-MLPs, SRNs and RAAMs, and the learning processes they use have strongly influenced the modular decomposition of connectionist parsing.

Hybridity

Only pure connectionist parsers such as those of $[34,21,36]$ attempt every aspect of the parse with neural networks and perform no symbol manipulation other than to interpret system outputs. When symbolic modules or processes are included, the result is a hybrid connectionist parser. In hybrid parsers the symbolic modules have commonly been assigned to tasks such as: i) short-term storage to hold intermediate parse states, current input states and full sentential parses (stacks and buffers); ii) long-term storage to hold structured knowledge about language, such as grammar rules, semantic networks, and tree structures; iii) symbol manipulation and communication to control the parsing process and coordinate interactions between (connectionist) modules. Pure connectionist parsers have attempted to avoid using symbolic modules by implementing connectionist architectures to act as stack memories and associative memories.

\section{Realism}


Converging Paths of Psycholinguistic Studies and Connectionist Parsing

A dominant theory of Human Sentence Processing (HSP) is a modular two-stage model [37] in which an independent processor provides an initial syntactic structure using only syntactic information. A second processing phase allows semantic and discourse information to be used to enable the parser to revise the initial structure. Lexical and syntactic processing are considered to be separate since it is hypothesised that lexical representations are retrieved whereas syntactic representations are constructed [38]. However, there is an increasing body of research that rejects this view and argues that HSP is a multiple constraint-satisfaction process that allows syntactic, semantic and discourse information to simultaneously interact (to varying degrees) during on-line processing [39,40,41]. The psycholinguistic evidence suggests that syntactic structure is projected from a mental lexicon enriched with word representations containing syntactic, semantic, and discourse information together with the frequency and probabilistic relationships that hold amongst them. Syntactically ambiguous sentences are commonplace. In the sentence, Gill saw the man with the telescope, we don't know whether the man or Gill has the telescope, and the two possiblities are syntactically distinct, suggesting the need for semantic intervention in the parsing process. Furthermore, psychologists have for decades accumulated evidence for the emergence of tentative interpretations of sentences occurring prior to a definitive parse [42].

Connectionist models can be employed as the processing mechanisms to develop and evaluate constraint-satisfaction theories $[39,43]$ and there is some evidence that connectionist research into syntactic processing is beginning to converge with 
psycholinguistic theories. Early localist parsing models were able to provide some account of human syntactic and semantic attachment preferences, whereby alternative interpretations remain active until they become incompatible with the rest of the sentence $[8,9,10]$. However, these techniques were based on a limited set of grammar rules. Stevenson's CAPERS model [44] extended localist parsing techniques further using a symbolic module, based on the principles defined in Chomsky's Government and binding (GB) Theory [45]; activation representing grammatical constraints is communicated between neighbouring units within a localist network that settles to a steady state of activations explicitly representing a parse tree. Stevenson demonstrated that CAPERS is able to establish long-distance dependencies between constituents without specially designed heuristics and addresses some of the psycholinguistic data reported by Stowe [46] and Nicol [47]. More recently, Stevenson and Merlo have used CAPERS to show that differences between unergative verbs (e.g. the horse raced past the barn fell) and unaccusative verbs (e.g. the butter melted in the pan was brown) at the lexical level significantly influence processing difficulty of sentences with ambiguities.

Although perhaps not directly motivated by the constraint-based theories per se, Hadley et al's [48] localist model supports the interaction of syntactic and semantic information. They proposed a self-organising, Hebbian-inspired, competitive network that was able to predict the coarse semantic features of the next word within a sentence for a small language domain. It was also able to predict syntactically key words, such as prepositions and relative pronouns. The model is significant since it generalised to structures of a complexity beyond that found in the training data and demonstrated 
strong syntactic systematicity in that it was able to recognise noun phrases in new (untrained) syntactic positions.

Connectionists have also exploited the advantages of distributed representations. St John and McClelland [49] presented a modular connectionist account of the multiple constraint-based model using two MLP-based networks. The model assigns thematic roles (e.g. agent and action) to predetermined syntactic phrases (constituents) of simple single-clause sentences based on syntactic and semantic constraints. It builds a single distributed representation of the sentence, called the sentence gestalt, which is adjusted as each constituent is processed. Given a word from a sentence it is able to reproduce the thematic role assigned to that word. Although evaluated using a very limited language, it was able to anticipate future constituents, and also learnt semantic regularities about the age of agents to predict appropriate objects. The main limitation of this approach is the inefficient localist representation used on input/output layers which provide little promise of scaling-up to work on realistic language sources.

Pearlmutter et al [50] proposed an FF-MLP-based model directly inspired by the constraint-based framework presented by MacDonald et al $[40,43]$. The localist input representation for nouns contained information such as subject animacy, voice and presence of a direct object. Verb representations contained information such as a unique indentifier for the verb and a set of simple semantic features. Given this input on the input layer the FF-MLP was expected to produce the appropriate verb argument structure on the output layer. After being trained on a set of 60 unambiguous verbs and associated argument structures extracted from the Wall Street Journal corpus, the FFMLP was tested on a modified training set. The training set was made ambiguous by 
removing the influence of voice and direct object inputs. The resulting behaviour of the system provided strong support for constraint-based theories. Pearlmutter et al reported that the frequency of argument structures found within the corpus directly influenced the processing of verb ambiguities.

\section{Realism Beyond Context-free Grammar}

Modular connectionist parsers that do not rely on a connectionist module such as RAAM to encode explicit structure have been able to capture realistic natural language structures from natural language corpora annotated with syntactic information. These parsers, for example those of Wermter and Weber [31], Lane and Henderson [19], and Tepper, Powell and Palmer-Brown [32,33] induce implicit grammars (that need never be expressed in the form of symbolic rules) via learning the underlying linguistic constraints contained in the annotations of corpus texts. To achieve this, connectionists have focused on using SRNs to perform tasks such as phrase boundary identification [31,32,33] and recognition of constituent dependencies [19], and on FF-MLPs to perform tasks such as phrase structure classification [32,33]. In these hybrid models, the symbols representing syntactic structure are represented by patterns which are processed and recognised by connectionist modules.

\section{Embedding and Cross-Serial Dependencies}

SRNs can process cross-serial dependencies (in which the correct interpretation of the syntactic role of a word or phrase is dependent on that of another word or phrase separated from it by a number of words) when there are local dependencies between all the intervening words [13], and SRNs can also carry information over embeddings without 
local dependencies if the number of intervening words was small [14]. In addition, Christiansen and Chater [51] have demonstrated that an SRN is able to process centreembedding and cross serial dependencies (albeit based on simple context-free grammars) in a manner analogous to the human performance found in psycholinguistic data, in which the ability to process embeddings and serial dependencies is limited. Miikkulainen, whose parser, CLAUSES [31], used a combination of four SRNs, found it easier to process tail recursion (e.g The woman blamed the man, who hit the girl, who blamed the boy) than relative clauses located in the middle of a sentence (e.g. The woman, who the boy, who the girl blamed hit, blamed the man) because the latter case requires longer sequences thus taxing the memory capacity further. The memory degradation in SRNs is due to previous input information degrading and ultimately being lost as more input is recursively encoded; and this type of degradation also applies to RAAMs [52], although the level of embedding a RAAM is capable of encoding can be reliably determined [53].

\section{Conclusion}

Connectionism is sometimes characterised as a unified approach to computation and cognition, with obvious strengths, such as the ability to acquire new information, and obvious weaknesses, such as limited recursive capabilities, and lack of systematicity. However, a review of connectionist parsing demonstrates that there are several related approaches, ranging from modular systems that mix symbolic processing with neural network processing, to systems that consist entirely of one or more neural networks and that learn to process syntax entirely from examples. Connectionist parsing is still negotiating its relationship with the world of symbols, but the current evidence supports a hybrid approach. The continued improvement in performance of connectionist parsers 
seems likely to be dependent on the discovery or development of more powerful neural

network learning processes and architectures, that can effectively assimilate the vast

quantity of complex information associated with human language processing ability.

\section{References}

Friederici, A. D. (2002) Towards a neural basis of auditory sentence processing. Trend. Cogn. Sci. 6(2), 78-84, February 2002.

Fodor J. A. (1983) Modularity of Mind, Cambridge, MA: MIT Press.

Marcus, G. (2001) The Algebraic Mind: Integrating Conectionism and Cognitive Science, MIT Press.

Selman, B. and Hirst, G. (1985) A rule-based connectionist parsing system. In Proceedings of 7th Annual Conference of the Cognitive Science, pp. 212-221.

Fanty, M. A. (1986) Context-free parsing with connectionist networks. In Proceedings of AIP Conference on Neural Networks for Computers, pp. 140-145.

Howells, T. (1988) VITAL: A connectionist parser. Proceedings of the 10th Annual Conference of Cognitive Science Society, pp. 18-25.

Santos, E. (1989) A massively parallel self-tuning context-free parser. Advances in Neural Info. Proc. Sys. 1, 537-544, Morgan Kaufmann.

Cottrell, G. W. and Small, S. (1983) A connectionist scheme for modeling word-sense disambiguation. Cognit. and Brain Theory 6, 89-120.

Waltz, D. and Pollack, J. (1985) Massively parallel parsing : A strongly interactive model of natural language interpretation. Cognit. Sci. 9, 51-74.

Cottrel, G. W. (1985) Connectionist parsing. In Proceedings of the 7th Annual Conference of the Cognitive Science Society.

Kwansy, S. C. and Faisal, K. (1993) Connectionism and determinism in a syntactic parser. In Proceedings of IEEE Conference on Connectionist Natural Language Processing.

Tepper, J. A. et al. (1995) Integrating symbolic and subsymbolic architectures for parsing arithmetic expressions and natural language sentences. In Proceedings of the $3^{\text {rd }}$ SNN Neural Network Symposium, Nijmegen University, September 1995.

Cleeremans, A. et al. (1989) Finite state automata and simple recurrent networks. Neural Comput. 1 (3), 372-381.

Elman, J. L. (1991) Distributed representations, simple recurrent networks, and grammatical structure. Machine Learn. 7 , 195-224.

Christiansen, M. H. and Chater, N. (1999) Connectionist natural language processing : the state of the art. Cognit. Sci. 23 (4), 417-437.

Lawrence, S. et al. (2000) Natural language grammatical inference with recurrent neural networks. IEEE Trans. on Knowl. and Data Eng. 12 (1), 126-140.

Servan-Schreiber, D. et al. (1991) Graded state machines: The representation of temporal contingencies in simple recurrent networks. Machine Learn. 7, 161-193.

Sells, P. et al. (1991) Foundational issues in natural language processing MIT Press, Cambridge, MA.

Lane, P. and Henderson, J., 1998, Simple Synchrony Networks : Learning to parse natural language with temporal synchrony variable binding. In Proceedings of International Conference on Artificial Neural Networks, Skovde, Sweden. Moisl, H. (1992) Connectionist finite state language processing. Conn. Sci. 4 (2), 67-91.

Miikkulainen, R. (1995) Subsymbolic parsing of embedded structures. In Computational Architectures Integrating Neural and Symbolic Processes, (Sun, R., ed), Kluwer Academic Publishers, Boston.

Mayberry, M. R. and Miikkulainen, R. (2000) Combining maps and distributed representations for shift-reduce parsing. In Hybrid Neural Systems (Wermter, S. and Sun, R., eds.), Springer Verlag, Heidelberg, pp. 144-157. Reilly, R. (1992) Connectionist technique for on-line parsing. Neural Nets. 3, 37-46.

Berg, G. (1992) A connectionist parser with recursive sentence structure and lexical disambiguation. Learning:Constructive and Linguistic, 32-37.

Zeng, Z. et al. (1994) Discrete recurrent neural networks for grammatical inference. IEEE Trans. on Neural Nets. 5(2), 320330 .

Martelli, M. (1992) Discrete Dynamical Systems and Chaos CRC Press.

Rodriguez, P. et al. (1999) A recurrent neural network that learns to count. Conn. Sci. 11(1), 5-40.

Wiles, J. et al. (2000) Representation beyond finite states: Alternatives to push-down automata. In A Field Guide to Dynamical Recurrent Networks, (Kolen, J. F. and Kremer, S. C., eds), IEEE Press, New York.

Sharkey, A. and Sharkey, N. (1992) Connectionism and natural language. In Connectionist Natural Language Processing (Sharkey, N. ed.), Part 20. pp. 1-10, Intellect.

Wermter, S. and Weber, V. (1994) Learning fault-tolerant speech parsing with SCREEN. In Proceedings of the Twelfth National Conference on Artificial Intelligence, Seattle.

Miikkulainen, R. (1990) A PDP architecture for processing sentences with relative clauses. Proceedings of the $13^{\text {th }}$ International Conference on Computational Linguistics, pp. 201-206.

Tepper, J. A. et al. (2001) Corpus-based connectionist parsing. In The Second Workshop on Natural Language Processing and Neural Networks (NLPNN2001) Tokyo, Japan, November 30, 2001.

Tepper, J. A. et al. (2002) A corpus-based connectionist architecture for large-scale natural language parsing, Accedpted for Connection Sci.

Ho, E. K. S. and Chan, L. W. (1997) Confluent preorder parsing of deterministic grammars. Conn. Sci. 9 (3), 269-293. 
Sharkey, N. and Sharkey, A. (1992) A modular design for connectionist parsing. In Twente Workshop on Language Technology 3 : Connectionism and Natural Language Processing, University of Twente, Enschede, The Netherlands, pp. 87-96.

Giles, C. L. et al. (1993) Learning and extracting finite state automata with second-order recurrent neural networks. Neural Comput. 4, 393-405.

Frazier, L. (1989) Against lexical generation of syntax. Lexical Representation and Process (Marslen-Wilson, W. D. ed.), MIT Press, Cambridge, Mass, 505-528.

Rayner, K. and Morris, R. (1992) Eye movement control in reading: Evidence against semantic preprocessing. J. of Experimental Psychol.: Human perception and performance, 18, 163-172.

Taraban, R. and McClelland, J. L. (1990) Parsing and comprehension: A multiple constraint view. In Comprehension Processes in Reading, (Balota, D. A., Flores d'Arcais, G. B. and Rayner, K., eds),Lawrence Erlbaum, Hillsdale, NJ, pp. 231-263.

MacDonald, M. et al (1994) Syntactic Ambiguity Resolution as Lexical Ambiguity Resolution. In Perspectives on Sentence Processing (Clifton, C., Frazier, L., and Rayner, K. eds) Hillsdale, N.J.: Lawrence Erlbaum Associates, pp. 123153

Tanenhaus, M. and Trueswell, J. (1995) Sentence Comprehension. In Speech, Language, and Communication, (Miller, J. and Eimas, P., eds) San Diego: Academic Press, pp. 217-262.

Greene, J. (1986) Language Understanding. Open University Press.

McDonald, M. C. et al. (1994) The lexical nature of syntactic ambiguity resolution. Psychol. Rev., 101, 676-703

Stevenson, S. (1994) Competition and recency in a hybrid network model of syntactic disambiguation. J. of Psycholing. Res. 23(4), 295-322.

Chomsky, N. (1981) Lectures on Government and Binding: The Pisa Lectures, Foris, Dordrecht.

Stowe, L. A. (1986) Parsing WH-constructions: Evidence for on-line gap location. Lang. and Cognit. Proc. 1, $227-245$.

Nicol, J. (1988) Coreference processing during sentence comprehension: Reactivating antecedents of empty categories during sentence processing, Unpublished Ph.D thesis, MIT.

Hadley, R. F. et al. (2001) Syntactic systematicity arising from semantic predictions in a Hebbian-competitive network. Conn. Sci.13 (1), 73-94.

. F. and Mc

Artif. Intel. 46, 217-258.

Cognit. Sci. 23, 157-206.
Pollack, J. B. (1990) Recursive Distributed Representations. Artif. Intel. 46, 77-105.

53 Callan, R. and Palmer-Brown, D. (1997) (S)RAAM : An analytical technique for fast and reliable derivation of connectionist symbol structure representations. Conn. Sci. 9, 139-159. 